\title{
Searches for diffuse fluxes of astrophysical neutrinos with the ANTARES telescope
}

\author{
Luigi Antonio Fusco ${ }^{1,2, a}$, Steffen Hallmann ${ }^{3}$, Sergio Navas ${ }^{4}$, and Federico Versari ${ }^{1,2}$ on behalf of \\ the ANTARES Collaboration \\ ${ }^{1}$ Dipartimento di Fisica e Astronomia dell'Università, Viale Berti-Pichat 6/2, 40127 Bologna, Italy \\ ${ }^{2}$ INFN - Sezione di Bologna, Viale Berti-Pichat 6/2, 40127 Bologna, Italy \\ ${ }^{3}$ Friedrich-Alexander-Universität Erlangen-Nürnberg, Erlangen Centre for Astroparticle Physics, Erwin- \\ Rommel-Str. 1, 91058 Erlangen, Germany \\ ${ }^{4}$ Departamento de Fisica Teorica y del Cosmos, Universidad de Granada, Av. Severo Ochoa, 18071 \\ Granada, Spain
}

\begin{abstract}
In this proceedings we report on the status of searches for diffuse fluxes of cosmic neutrinos with the ANTARES neutrino telescope data. A complete overview of diffuse neutrino searches will be given, together with the search for a neutrino emission from regions such as the Fermi Bubbles or the Galactic Plane. A non-significant, though intriguing, excess of events above the atmospheric background is observed in all-sky analysis both for the track and shower channels.
\end{abstract}

\section{Introduction}

Cosmic Rays (CR) produced at astrophysical objects such as Supernova Remnants, Active Galactic Nuclei or Gamma Ray Bursts can interact in the interstellar medium and produce neutrinos. A diffuse flux of cosmic neutrinos is expected from unresolved individual sources or CR propagation in the Universe. The energy spectrum of these neutrinos should be similar to that of primary CRs and flatter than the observed atmospheric neutrino background.

The ANTARES detector [1] is at present the largest neutrino telescope in the Northern Hemisphere, located at a depth of $2475 \mathrm{~m}$ in the Mediterranean Sea, $40 \mathrm{~km}$ off-shore Toulon, France and has been continuously operated since 2007. It consists of 885 photomultiplier tubes (PMTs), distributed along 12 mooring lines anchored to the sea bed and kept taut by top buoys. It detects the products of all-flavour neutrino interactions by collecting the Cherenkov photons emitted by relativistic charged particles. Charged current (CC) $v_{\mu}$ interactions, where a straight muon track is produced, can be reconstructed in direction with a good pointing accuracy [2]. All-flavour neutral current (NC) interactions, as well as $\mathrm{CC} v_{e}$ and $v_{\tau}$ interactions are as well reconstructed with good accuracy, thanks to the optical properties of water [3].

The main background in searches for diffuse fluxes of a cosmic signal is given by atmospheric neutrinos and muon bundles produced in CR air showers. To reduce the background from atmospheric

a e-mail: lfusco@bo.infn.it 
muons, only upward-going events are selected since only neutrinos can traverse the Earth. Nonetheless, wrongly reconstructed atmospheric muons can survive this directional selection: a proper cut on the reconstruction quality parameter can reduce this background. The background from atmospheric neutrinos, whose spectrum is softer, is reduced by a cut on energy estimators [4].

The IceCube Collaboration has reported [5] the observation of an all-flavour diffuse and isotropic excess of high energy neutrinos, not compatible with atmospheric expectations. Different approaches are possible to analyse this signal and the efforts made by the ANTARES collaboration are presented in the following sections. Blinded analysis procedures have been followed, with only $10 \%$ of data available for tests and data/Monte Carlo comparison. The good agreement between data and Monte Carlo allowed for a final cut choice based on simulations.

\section{Diffuse flux analyses}

The analysis of diffuse $v_{\mu}$ fluxes uses track-like events in the complete ANTARES data-sample (20072015). The equivalent livetime is 2451 days. Most of the down-going atmospheric muon events are rejected applying a preliminary cuts on the reconstructed zenith angle $\left(\theta_{z e n}>90^{\circ}\right)$ and on the angular error estimation $\beta\left(\beta<0.5^{\circ}\right)$. To further reduce the atmospheric background combined cut on reconstruction quality parameter, the reduced $\log$-likelihood $\Lambda$, and on the number of PMT-signals (hits) related to the reconstructed track, $\mathrm{N}_{h i t}$, has been applied, thus reducing the muon background level well below $1 \%$.

Atmospheric neutrinos can be rejected by applying an energy-related cut, based on the estimation of the muon energy using an Artificial Neural Network [6]. The optimal cut on this variable is chosen by the Model Rejection Factor (MRF) procedure [7] to give the best sensitivity flux. After the final cut $13.5_{-4}^{+3}$ events are expected from the background and 3 events should be produced by an IceCube-like signal [5]. After unblinding and applying the energy selection, 19 events are found in data, as shown in figure 1 - left.

All events reconstructed with the shower algorithm of [3] are considered in the cascade analysis. Data from 2007 to 2013, with a total livetime of 1451 days, have been used. An event pre-selection is done using a containment cut on the vertex position and requiring events to be upward-going. Various quality parameters and track-distriminating quantities are used to reduce the background from atmospheric muons. Finally the MRF optimisation is done on the reconstructed shower energy. The optimal cut is found to be $\mathrm{E}_{\text {shower }}>30 \mathrm{TeV}$. With this selection, $5 \pm 2$ background events are expected, and an IceCube-like signal flux would correspond to about 1.5 cosmic events added to the atmospheric expectations. 7 events are observed in data after unblinding as shown in figure 1 - right.

By construction there is no overlap between the track and the shower samples, since an anti-track selection is applied in the shower selection chain. The samples can be combined and, overall, 26 events are observed in data over an expected background of $18 \pm 5$. Since the excess is not statistically significant $(<1.5 \sigma)$, an upper limit at $90 \%$ confidence level (c.l.) can be computed according to [8] and is shown in figure 2. This estimation of the upper limit does not take into account yet systematic effects, mainly related to the uncertainties of the optical module efficiency and water properties.

\section{Special regions}

Southern-sky areas showing a diffuse and enhanced emission of $\gamma$-rays are suitable targets to search for a diffuse neutrinos emission, since ANTARES has large exposure to these parts of the sky. For this kind of analysis, an on/off-zone procedure has been followed: the background is estimated from regions of the sky with the same exposure as the expected signal regions and is then compared to the 

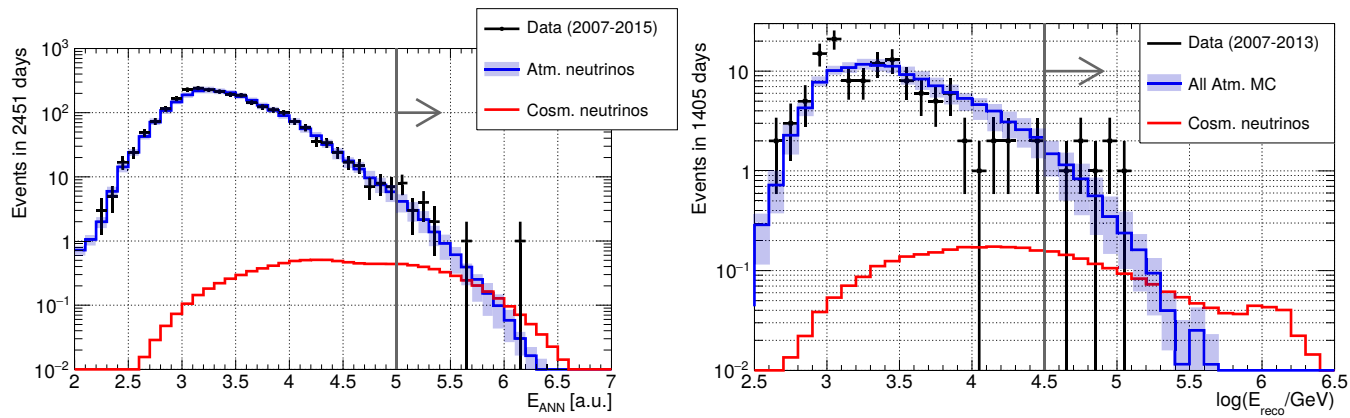

Figure 1: Left: energy estimator distribution for the track channel after the event selection chain. Right: same for the shower channel. Red lines indicate the cosmic neutrino expectation for the IceCube cosmic flux, while blue lines represent the contribution from atmospheric events, with the shaded areas providing an estimate of the related uncertainties. The gray lines and arrow show the chosen energy-related cut.

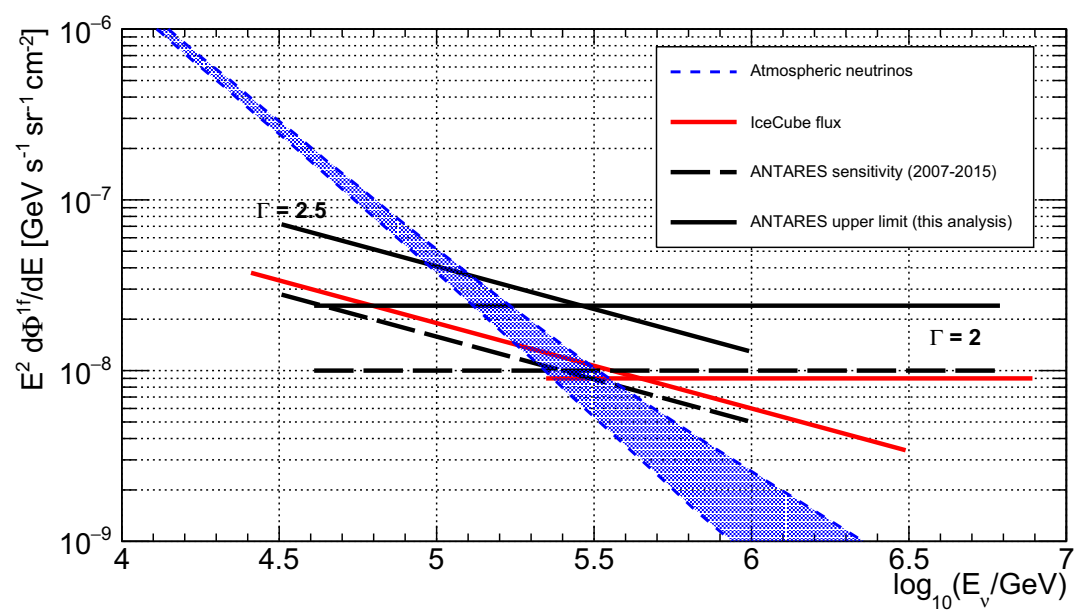

Figure 2: Upper limit for the combination of the two analyses (solid black lines) for the two spectral indices considered ( $\Gamma=2$ and 2.5) compared to the final sensitivity (black dashed lines) expected when including the whole available livetime. Also shown in red the best fit from IceCube analyses for the two spectral hypotheses and the contribution from atmospheric neutrinos (blue shaded area).

observation in the signal region. In the absence of signal, the observed number of events in on and off-zones should be statistically compatible.

\subsection{Fermi Bubbles}

Fermi telescope data $[9,10]$ have revealed the presence of two giant $\gamma$-ray emission regions above and below the Galactic Plane. If hadronic mechanisms are responsible for the production of such a signal, 

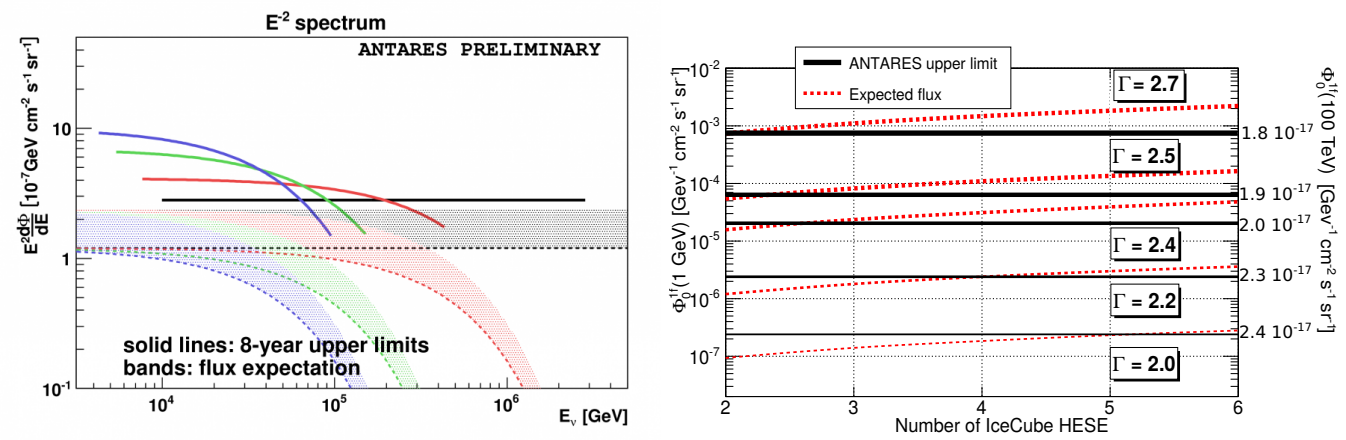

Figure 3: Left: upper limit calculation for neutrino fluxes from the Fermi Bubbles for different energy cut-offs $(\infty, 100 \mathrm{TeV}, 50 \mathrm{TeV}, 10 \mathrm{TeV}$ - black, red, green and blue lines). Shaded areas are for theoretical predictions with the various cuf-offs. Right: upper limits and sensitivity for Galactic Plane analyses from ANTARES for different spectral indexes compared to a flux producing a certain number of IceCube High Energy Starting Events (3 years sample) [16].

diffuse neutrino emissions are expected from these regions with various possible energy cut-offs from few to some hundreds of TeV [11].

Data collected in the $v_{\mu}$ CC channel with the ANTARES telescope from 2007 to 2015 are considered in this analysis. The event selection is based on cuts on the quality of up-going reconstructed tracks and, as in the previous analysis, on the energy estimator based on the ANN method. The final selection was obtained by minimizing the MRF and optimizing for a neutrinos flux with a $100 \mathrm{TeV}$ cut-off. After the unblinding of the on-zone, 28 events are observed, while 19.7 are expected, on average, from the off-zones. The significance of this excess can be estimated, following the prescription of [12], as $1.5 \sigma$, and $90 \%$ c.l. upper limits, computed as in [8] are shown in figure 3.

\subsection{Galactic Plane}

A diffuse neutrino flux is expected from the decays of charged mesons produced in CR interactions in the interstellar medium in the Galactic Plane [13]. The corresponding emission from neutral mesons is clearly visible in $\gamma$-ray observation of the sky [14]. Different models for the CR propagation are proposed and can be tested using neutrino data.

In this case, as in the case of the diffuse analysis, only muon data from 2007 to 2013 have been analyzed [15] following the MRF procedure to set an optimal cut on the area of the on-zone and on the quality and energy of reconstructed tracks. The 9 selected off-zones show on average 3.5 events, and 2 are observed in the on-zone after unblinding. Correspondingly, 90\% c.l. upper limits are put (equal to the sensitivity) on the possible contribution from the Galactic Plane to the IceCube signal from [16] as shown in figure 3.

\section{Conclusions}

Data collected by the ANTARES telescope from 2007 to 2015 have been analysed and various searches have produced interesting results regarding the diffuse neutrino signal observed by the IceCube collaboration. An intriguing excess of events is reported in both the channels of all-sky searches, 
even though still compatible with the expectations from atmospheric backgrounds. Stringent limits are also being put on the possible galactic origin of the IceCube signal, lowering the possible number of IceCube HESE events origintating from the Galactic Plane down to 2 for the three years sample.

More data are being analysed and the expected sensitivity for the whole data sample in all-sky searches is close to the observed IceCube flux for all the considered scenarios. Further improvements are possible especially in the shower channel, for which the event selection chain can be optimised in order to reduce the background from atmospheric events. This is also true for special region searches, since the angular resolution of ANTARES in the shower channel is of the order of few degree and an enhancement of the detector performance is expected when adding also cascade-like events in the analysis [17].

\section{References}

[1] M. Ageron et al., Nucl. Instr. and Meth. A 656: 11 (2011).

[2] J. Barrios Martí et al., Proceedings of the $34^{\text {th }}$ ICRC, The Hague, Netherlands, arXiv: 1510.04508 (2015).

[3] T. Michael et al., Proceedings of the $34^{\text {th }}$ ICRC, The Hague, Netherlands, arXiv:1510.04508 (2015).

[4] S. Adrián-Martínez et al. EPJ C 73: 2606 (2013).

[5] M.G. Aartsen, et al., Astrophys. J. 809: 98 (2015).

[6] J. Schnabel et al., Nucl. Instr. and Meth. A 725: 106-109 (2013).

[7] G.C. Hill and K. Rawlins, Astropart. Phys. 19: 393 (2003).

[8] G.J. Feldman and R.D. Cousins, Phys. Rev. D 57: 3873-3889 (1998).

[9] M. Su et al., Astrophys. J. 724: 1044 (2010).

[10] M. Ackermann et al., Astrophys. J. 793: 64 (2014).

[11] R. M. Crocker and F. Aharonian, Phys. Rev. Lett. 106: 101102 (2011).

[12] T. P. Li and Y. Q. Ma, Astrophys. J. 272: 317 (1983).

[13] D. Gaggero et al., ApJ Letters 815: L25 (2015).

[14] M. Ackermann et al., Astrophys. J. 750: 3 (2012).

[15] S. Adrián-Martínez et al. Phys. Lett. B 760: 143-148 (2016).

[16] M.G. Aartsen et al., Phys. Rev. Lett. 113:101101 (2014).

[17] T. Grégoire et al. Proceedings of the $27^{\text {th }}$ Neutrino Conference, London, UK (2016). 